\title{
A Review of Proximity Gettering Technology for CMOS Image Sensors Using Hydrocarbon Molecular Ion Implantation
}

\author{
Kazunari Kurita, ${ }^{*}$ Takeshi Kadono, Ryousuke Okuyama, Satoshi Shigematsu, \\ Ryo Hirose, Ayumi Onaka-Masada, Yoshihiro Koga, and Hidehiko Okuda
}

SUMCO, 1-52 Kubara, Yamashiro-cho, Imari-shi, Saga 849-4256, Japan

(Received January 28, 2019; accepted April 15, 2019)

Keywords: CMOS image sensors, gettering technique, metallic impurity, hydrocarbon molecular ion implantation, white spot defects, dark current, image lag, dark current spectroscopy

We developed a high-gettering-capability silicon wafer for advanced CMOS image sensors using hydrocarbon molecular ion implantation. We found that this novel silicon wafer has an extremely high gettering capability for metal, oxygen, and hydrogen impurities during the CMOS device fabrication process. We also found that the white spot defect density of a hydrocarbon-molecular-ion-implanted CMOS image sensor was substantially lower than that of a CMOS image sensor without hydrocarbon molecular ion implantation. This indicates that the novel silicon wafer helped improve device performance parameters such as white spot defect density and dark current. We believe that this wafer will be beneficial in the design of silicon wafers for advanced CMOS image sensor fabrication.

\section{Introduction}

CMOS image sensors have become widely used in Internet of Things (IoT) devices such as smart phones, smart watches, and personal computer tablets. ${ }^{(1)}$ The consumer market for such devices strongly requires a wide dynamic range, highly sensitive imaging, and high-speed image data processing for fabricating high-performance CMOS image sensors. ${ }^{(2)}$ However, there are serious technical issues (Fig. 1) involved in fabricating them.

The first important issue is metallic impurity contamination in the device active region during CMOS device fabrication process steps such as high-temperature rapid thermal treatment and plasma etching treatment. ${ }^{(3,4)}$ Metallic impurities form deep energy level defects in the silicon band gap. ${ }^{(5,6)}$ These defects strongly affect device electrical performance parameters such as white spot defect density, recombination lifetime, dark currents, and gate oxide breakdown voltage. ${ }^{(7-9)}$ Thus, CMOS image sensor manufacturers make much effort to eliminate metallic impurities from the device active region using gettering techniques. Intrinsic gettering (IG) is the most popular gettering technique in the semiconductor device fabrication process. ${ }^{(10)}$ This technique involves gettering metallic impurities by using an

*Corresponding author: e-mail: k-kurita@sumcosi.com https://doi.org/10.18494/SAM.2019.2313 


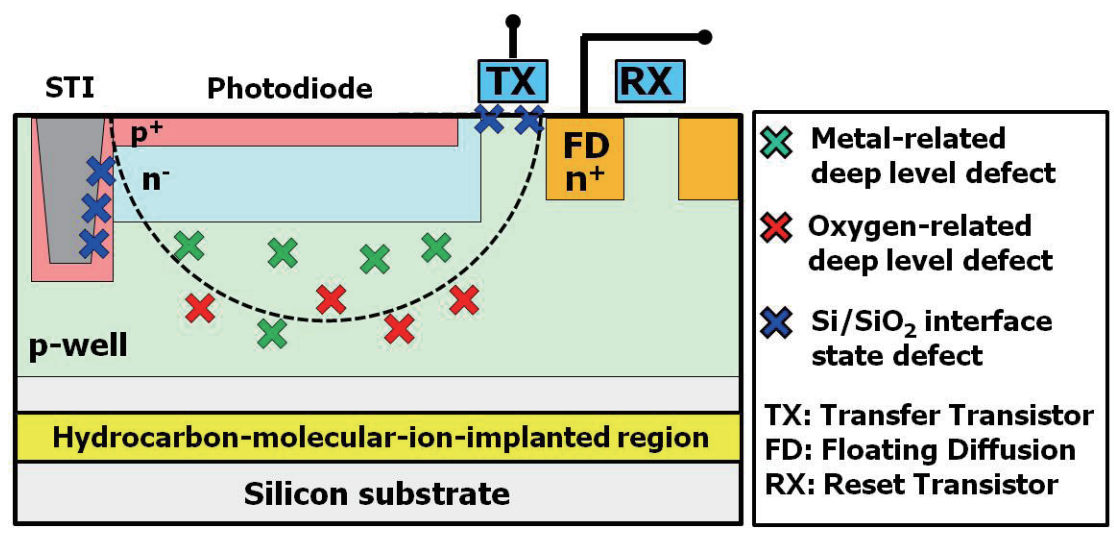

Fig. 1. (Color online) Technology issues for CMOS image sensor fabrication. Modified from Kurita et al. ${ }^{(26)}$ Copyright (2016) The Japan Society of Applied Physics.

oxygen precipitation growth process during the device heat treatment..$^{(11,12)}$ For advanced CMOS devices, thermal heat budgets are a much lower temperature and a shorter duration time year by year. Thus, oxygen precipitation is not enough for the growth of oxygen precipitate nucleation center, which can act as gettering sinks for metallic impurities during the device fabrication process. Thus, IG becomes unsuitable for fabricating advanced CMOS image sensors. Another gettering solution is high-energy ion implantation induced by damageinduced defects for gettering sinks in the silicon substrate. ${ }^{(13,14)}$ It is well known that these defects induce secondary extended defects such as dislocations and dislocation loops after the device heat process. The secondary extended defects can act as extremely effective gettering sinks for metallic impurities in the silicon substrate. ${ }^{(15-19)}$ However, these defects expand to the top surface region of the silicon substrate, which makes it extremely difficult to grow epitaxial silicon in the region. Thus, the high-energy ion implantation technique is not a good gettering technical solution for fabricating advanced CMOS image sensors.

The second important issue is oxygen out-diffusing to the device active region from a Czochralski (CZ)-grown silicon substrate during the CMOS device heat process. The CZgrown silicon crystals produced by a crystal growth process using a quartz crucible contain high oxygen impurities in bulk silicon. ${ }^{(12)}$ The oxygen impurities form oxygen-related deep energy level defects in the silicon band gap, which potentially can act as potential pockets and barriers in the device active region. ${ }^{(1)}$ These defects strongly affect device electrical performance factors such as image lag characteristics. ${ }^{(2)}$ Thus, CMOS image sensor manufacturers strongly demand the control of oxygen impurities out-diffusing to the device active region from a CZ-grown silicon substrate during the device fabrication process. Thus, the solution to the above issue is the use of CZ-grown silicon wafers with low oxygen concentration. The out-diffusion amount of such wafers decreases during the device fabrication process. However, the mechanical strength of the silicon wafers is much lower than that of conventional CZ-grown silicon crystals. Thus, it is extremely difficult to use these wafers for CMOS image sensor mass production.

The third important issue is the carrier generation from interface state defects such as the presence of transistor gate oxide in the $\mathrm{Si} / \mathrm{SiO}_{2}$ interface region, local oxidation in silicon (LOCOS), 
shallow trench isolation (STI), and deep trench isolation (DTI). ${ }^{(1,2)}$ These carrier generations strongly affect electrical device performance parameters such as the levels of fixed pattern noise, flicker noise, and random telegram signal (RTS) noise in CMOS image sensors. ${ }^{(20,21)}$ Therefore, CMOS image sensor manufacturers make strong effort to reduce the interface state density using low- temperature hydrogen annealing treatment. This treatment decreases the $\mathrm{Si} /$ $\mathrm{SiO}_{2}$ interface state density of $\mathrm{Pb}$ and $\mathrm{E}$ ' centers by using a hydrogen-terminated process. ${ }^{(22)}$ However, the three-dimensional stacked CMOS image sensor (3D-CIS) fabrication process has been using multilayer deposition on the device surface fabricated by using the atomic layer deposition technique. ${ }^{(22)}$ The device surface deposits on multiple dielectric layers are performed before low-temperature hydrogen annealing. Most of the hydrogen atoms are trapped in the multiple dielectric layers during the low-temperature hydrogen annealing. ${ }^{(23)}$ The hydrogen does not diffuse to the device active region in the CMOS image sensor. The hydrogen amount is insufficient for process-induced defect (interface state defect) passivation by hydrogen. Therefore, CMOS image sensor manufacturers have been demanding an alternative to lowtemperature hydrogen annealing for effectively decreasing the process-induced defect (interface state defect) density in the device active region. The CMOS image sensor manufacturers strongly require silicon wafer solutions to address the above three technical issues for achieving a high performance in CMOS image sensor manufacturing.

How can we address these technical issues associated with CMOS image sensor manufacturing? The gettering technique is considered to be the most critical solution of the above issues with advanced CMOS image sensor manufacturing. The considered gettering technique forms gettering sinks near the device active region through ion implantation and can be called proximity gettering. The advance CMOS device manufacturing thermal budget tended to be lower than that in the conventional CMOS device manufacturing thermal budget. Thus, when slowly diffused metallic impurities such as Fe, W, and Mo do not diffuse to the gettering sinks in the epitaxial silicon wafer substrate such as IG technique during the low temperature device heat process, it is extremely difficult to obtain a high gettering capability for slowly diffused metallic impurities using a conventional IG technique. Therefore, we have been developing an alternative silicon wafer gettering technology solution for advanced CMOS image sensor fabrication using hydrocarbon molecular ion implantation. ${ }^{(24)}$ This novel proximity gettering technology realized the formation of a gettering sink near the device active region. We call it a hydrocarbon-ion-implanted silicon wafer. We found that this novel silicon wafer has three unique gettering characteristics for the improvement of advanced CMOS image sensors. ${ }^{(25)}$ First, a hydrocarbon-molecular-ion-implanted silicon wafer has a high gettering capability for metallic impurities. ${ }^{(26)}$ Second, with respect to the oxygen out-diffusion barrier effect, this wafer can control oxygen out-diffusion to the device active region from the CZgrown silicon substrate during the device fabrication process. ${ }^{(27)}$ Third, it produces a hydrogen passivation effect for process-induced defects (interface state defects) in CMOS image sensors. The hydrogen was gettered in the hydrocarbon molecular ion projection range. ${ }^{(28)}$ We expected that the hydrogen would out-diffuse to the device active region from the projection range during the CMOS device fabrication process. ${ }^{(29)}$ This phenomenon will have a passivation effect on process-induced defects such as those in the $\mathrm{Si} / \mathrm{SiO}_{2}$ interface states. ${ }^{(30)}$ 
In this review article, the main objective is to demonstrate the metal, oxygen, and hydrogen impurity gettering capability of the hydrocarbon-molecular-ion-implanted silicon wafer using the CMOS image sensor fabrication process. Furthermore, we investigate the effect of white spot defects on the CMOS image sensor with and without hydrocarbon molecular ion implantation for the first time. Few previous studies of white spot defects were evaluated by CMOS image sensors using the proximity gettering technique.

Therefore, we demonstrate that the novel silicon wafer has a high gettering capability and substantially decreases the white spot defect density as evaluated by dark current spectroscopy. It is extremely important to understand the gettering properties of the hydrocarbon-molecularion-implanted silicon wafer for understanding advanced CMOS image sensors from the viewpoints of fundamental material science and silicon wafer gettering design for CMOS image sensor fabrication.

We propose a solution to CMOS image sensor technical issues for designing silicon wafer gettering using hydrocarbon molecular ion implantation to achieve a high performance in CMOS image sensor manufacturing.

\section{Materials and Methods}

\subsection{Production concept of hydrocarbon-molecular-ion-implanted silicon wafer}

Figure 2 shows the production concept of hydrocarbon-molecular-ion-implanted epitaxial silicon wafers. First, the hydrocarbon-molecular-ion-implanted silicon substrate top surface

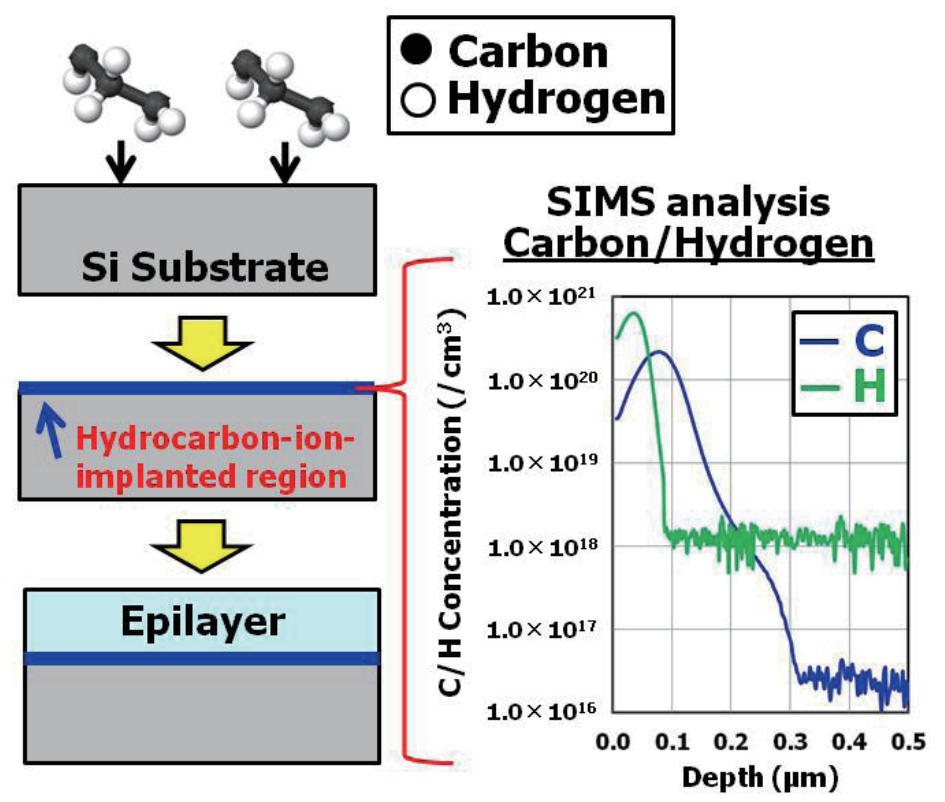

Fig. 2. (Color online) Production concept of hydrocarbon-molecular-ion-implanted epitaxial silicon wafers. Modified from Kurita et al. ${ }^{(26)}$ Copyright (2016) The Japan Society of Applied Physics. 
without an oxidation film and additional recrystallization heat treatment using a hydrocarbon molecular ion implanter (model CLARIS) were provided by Nissin Ion Equipment, Japan. ${ }^{(31-33)}$ The hydrocarbon molecular ion forms a carbon and hydrogen projection range after implantation. Second, the epitaxial silicon layer was deposited on the silicon substrate surface by chemical vapor deposition. Thus, the production concept of this novel silicon wafer is extremely simple. ${ }^{(24)}$

Figure 3 shows the SIMS depth profile measured on hydrocarbon-molecular-ion-implanted epitaxial silicon wafers after heat treatment. The hydrocarbon molecular ion implantation projection range forms carbon and hydrogen implantation elements. In this projection range, the carbon peak concentration is $2 \times 10^{20} \mathrm{~cm}^{-3}$ and the hydrogen peak concentration is $8 \times 10^{20}$ $\mathrm{cm}^{-3}$. Moreover, the carbon concentration in this projection range is three orders of magnitude higher than the solid solubility of carbon impurities in the silicon crystal after heat treatment.

\subsection{Sample preparation and evaluation techniques}

The experimental samples used in this study were (100) phosphorus-doped CZ silicon single crystals of $750 \mu \mathrm{m}$ thickness. Their resistivity was $10 \Omega \mathrm{cm}$ and their oxygen concentration was 1.4 $\times 10^{8}$ atoms $/ \mathrm{cm}^{3}$ (old ASTM). The sample wafers were subjected to $\mathrm{C}_{3} \mathrm{H}_{5}$ hydrogen molecular ion implantation of $80 \mathrm{keV}$ to a dose of $1 \times 10^{15} \mathrm{~cm}^{2}$. Their resistivity was also $10 \Omega \mathrm{cm}$ and the epitaxial layer grown by chemical vapor deposition was $5 \mu \mathrm{m}$ in thickness. The metallic impurity contaminated hydrocarbon-ion implanted epitaxial silicon wafers were fabricated by surface spin coating with a metallic-impurity-contaminated SC1 and SC2 acid solution. The nickel and copper surface metallic impurities contamination concentration at a level of $1 \times$ $10^{13}$ atoms $/ \mathrm{cm}^{2}$ on their surfaces. After the metallic impurity contamination, the wafers were annealed by heat treatment. The metal, oxygen, and hydrogen impurities of diffusion and the depth profile in the $\mathrm{C}_{3} \mathrm{H}_{5}$ molecular ion implantation projection range $\left(\mathrm{C}_{3} \mathrm{H}_{5}\right.$ ions at $80 \mathrm{keV}$ to

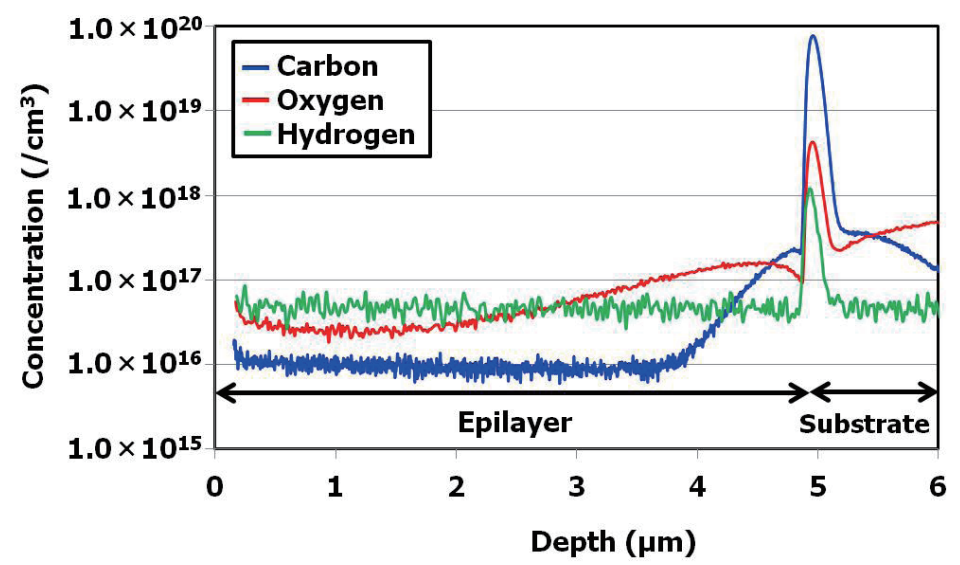

Fig. 3. (Color online) SIMS depth profile of hydrocarbon-molecular-ion-implanted epitaxial silicon wafers after epitaxial layer growth process. Modified from Kurita et al. ${ }^{(26)}$ Copyright (2016) The Japan Society of Applied Physics. 
a dose of $1 \times 10^{15} \mathrm{~cm}^{-2}$ ) were measured by secondary ion mass spectroscopy (SIMS) and atom probe tomography (APT) depth analysis before and after the CMOS heat treatment. The APT tool is very efficient in obtaining a $3 \mathrm{D}$ impurity distribution in semiconductor materials with an atomic-scale spatial resolution by CAMECA's LEAP 5000 atom probe microscopy. ${ }^{(34)}$

\section{Results}

\subsection{Metallic impurity gettering capability after heat treatment}

Figure 4 shows the nickel and copper metallic impurities gettered in the hydrocarbon molecular ion implantation projection range after intentional surface metal contamination and diffusion heat treatment. These results indicate that the metallic impurities diffused to the hydrocarbon molecular ion projection range during heat treatment. Thus, the metallic impurities were gettered in the hydrocarbon molecular ion projection range. This indicates that the novel silicon wafer has a high gettering capability for metallic impurities. ${ }^{(26,30)}$

\subsection{Oxygen out-diffusion barrier effect during device heat treatment}

Figure 5 shows the SIMS depth profiles of oxygen impurities measured on the epitaxial silicon wafers with and without hydrocarbon molecular ion implantation after device heat treatment. The oxygen impurities out-diffused to the silicon epitaxial layer from the CZ-silicon grown substrate during the device heat treatment. For a hydrocarbon-molecular-ion-implanted epitaxial silicon wafer, the oxygen impurities were gettered in the hydrocarbon ion implantation projection range during the device heat treatment. The hydrocarbon ion implantation projection range proved to be an effective gettering sink for controlling out-diffused oxygen impurities from a CZ-grown silicon substrate. This result indicates that the oxygen out-diffusion

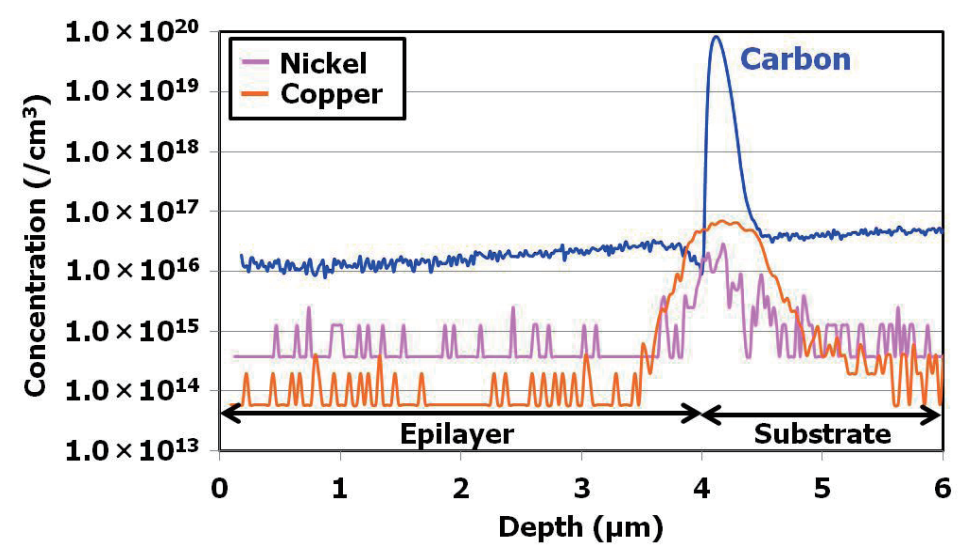

Fig. 4. (Color online) SIMS depth profile of hydrocarbon-molecular-ion-implanted epitaxial silicon wafer after metallic impurity diffusion heat treatment. This epitaxial silicon wafer sample was $\mathrm{C}_{3} \mathrm{H}_{5}$ hydrocarbon-molecularion-implanted at $80 \mathrm{keV}$ to a dose of $1 \times 10^{15} \mathrm{~cm}^{-2}$. Modified from Kurita et al. ${ }^{(26)}$ Copyright (2016) The Japan Society of Applied Physics. 


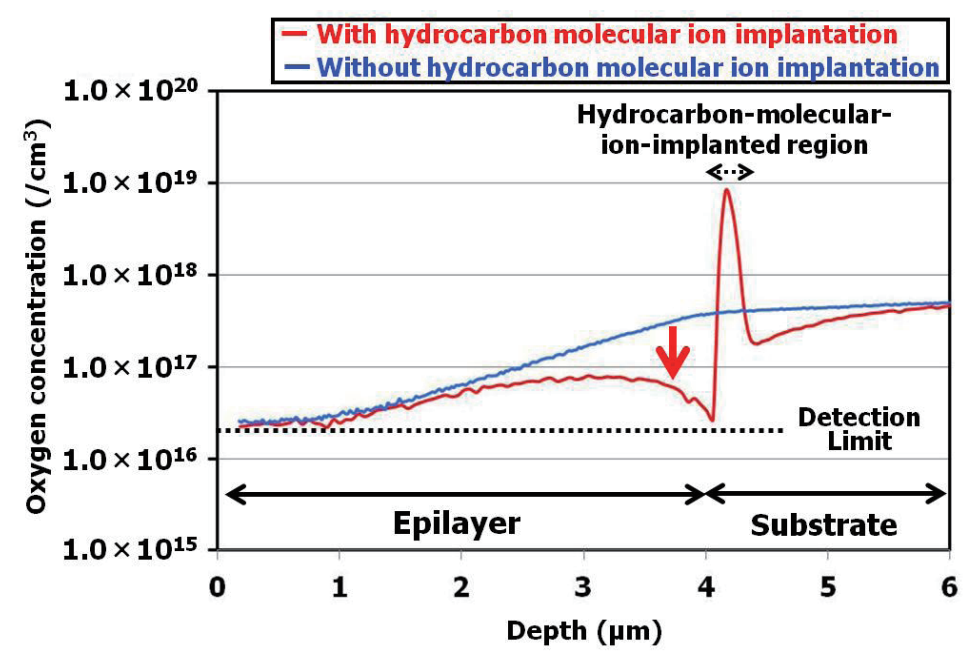

Fig. 5. (Color online) SIMS depth profiles of oxygen impurities in epitaxial silicon wafers with and without hydrocarbon molecular ion implantation after CMOS image sensor fabrication process. Modified from Kurita et al. ${ }^{(25)}$ Copyright (2015) The Japan Society of Applied Physics.

magnitude of a hydrocarbon-ion-implanted epitaxial silicon wafer is one order lower than that of an epitaxial silicon wafer without hydrocarbon molecular ion implantation. Thus, the hydrocarbon-ion-implanted epitaxial silicon wafer has a higher gettering capability for oxygen impurities than the epitaxial silicon wafer without hydrocarbon molecular ion implantation.

\subsection{Hydrogen gettering effect during device heat treatment}

The hydrocarbon molecular ion implantation forms a hydrogen and carbon impurity projection range in the silicon wafer top surface region as shown in Fig. 2. The position of each ion implantation element peak depends on the hydrocarbon mass number and acceleration ion implantation energy. The acceleration energy of the hydrogen element is $2 \mathrm{keV}$ and that of the carbon element is $22 \mathrm{keV}$. Thus, peak position hydrogen forms a silicon wafer top surface region. Figure 6 shows the SIMS depth profile of the hydrogen implantation element in the projection range measured on a hydrocarbon-molecular-ion-implanted silicon wafer before epitaxial growth. Figure 7 shows the SIMS depth profile of hydrogen in the projection range of the hydrocarbon-molecular-ion-implanted silicon wafer after epitaxial growth. The hydrogen concentration substantially decreased in the projection range after epitaxial growth.

This is a very interesting experimental data, because hydrogen is generally a light element and extremely easily diffuses in the silicon crystal bulk during the heat treatment. ${ }^{(35-38)}$ Thus, it does not remain in the silicon crystal bulk after the heat treatment. However, for hydrocarbon molecular ion implantation, it remained in the cross of the hydrogen and carbon projection range after the epitaxial growth as shown in Fig. 3.

Why did hydrogen remain in the hydrocarbon ion implantation projection range? The reason is that the cross of the hydrogen and carbon projection range forms stress and strain in the projection range. Thus, hydrogen is gettered by this stress and strain after the epitaxial growth 


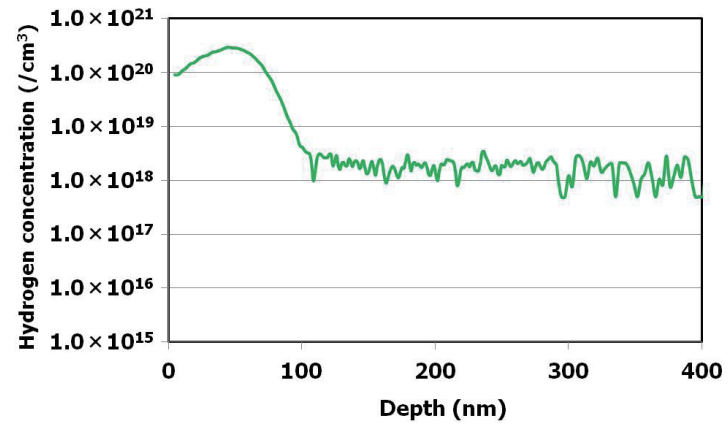

Fig. 6. (Color online) SIMS depth profile of hydrogen impurities in silicon wafers with hydrocarbon molecular ion implantation before epitaxial growth process. ${ }^{(26)}$ Copyright (2016) The Japan Society of Applied Physics.

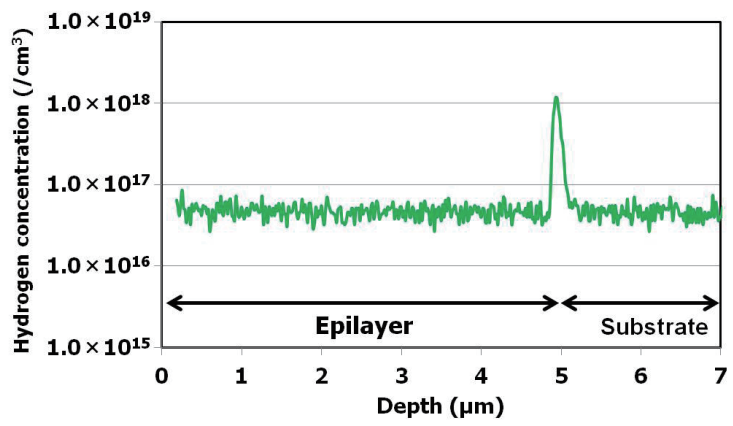

Fig. 7. (Color online) SIMS depth profile of hydrogen impurities in silicon wafers with hydrocarbon molecular ion implantation after epitaxial growth process. Modified from Kurita et $a l{ }^{(26)}$ Copyright (2016) The Japan Society of Applied Physics.

process. This indicates that a hydrocarbon-ion-implanted epitaxial silicon wafer has a high gettering capability for hydrogen impurity after the heat treatment.

\subsection{Gettering capability of hydrocarbon-molecular-ion-implanted epitaxial silicon wafer fabricated by CMOS image sensor using dark current spectroscopy}

We consider the gettering capability of a hydrocarbon-molecular-ion-implanted epitaxial silicon wafer using a CMOS device fabrication line. We evaluated the white spot defect densities of CMOS image sensors with and without hydrocarbon molecular ion implantation using dark current spectroscopy (DCS). ${ }^{(39)}$ DCS is an extremely powerful metallic impurity contamination analysis tool for charge-coupled devices (CCDs) and CMOS image sensors. ${ }^{(40)}$ This technical principle behind it is very similar to that of deep-level transient spectroscopy (DLTS). ${ }^{(41)}$ It is well known that DLTS is an extremely useful metallic impurity contamination analysis tool and that it is used to measure transient capacitance temperature dependence in metal Schottky barrier-junction or pn-junction capacitor diodes. DLTS makes it possible to determine the concentration of metallic-impurity-related defects and the defect activation energy. It also makes it possible to capture a cross section in the silicon band gap. Thus, DLTS can identify metallic impurities in silicon crystal bulk. However, it cannot measure DLTS signals using conventional CMOS device structures such as MOS-FET. ${ }^{(42)}$ DLTS signal measurements can only be applied to Schottky barriers or pn-junction diodes. In contrast, DCS can measure metallic-impurity-related electrical signals using a CMOS device structure. Moreover, the metallic impurity detection limit of DCS is two or three orders of magnitude higher than that of DLTS. Thus, solid-state image sensor manufacturers have used DCS to analyze metallic impurity contamination in CCD and CMOS image sensors. ${ }^{(4)}$

Figure 8 shows the DCS spectra of a CMOS image sensor with and without hydrocarbon molecular ion implantation after the device fabrication process. ${ }^{(43)}$ These spectra were measured from a CMOS image sensor using CMOS image sensor pixel architecture, which was 


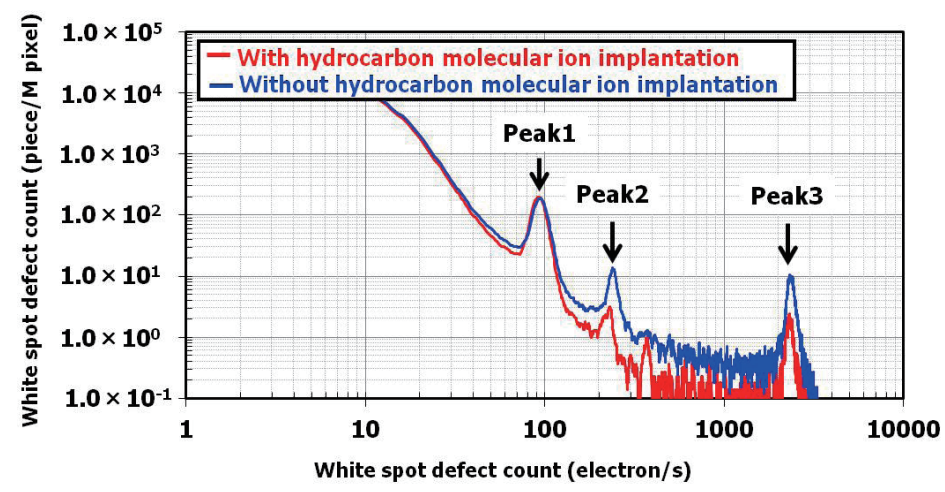

Fig. 8. (Color online) White spot defect counts of CMOS image sensors with and without hydrocarbon molecular ion implantation after CMOS image sensor fabrication process. ${ }^{(43)}$

a four-transistor-type pinned photodiode fabricated using a CMOS device process line. ${ }^{(44,45)}$ We found that the DCS spectra of the CMOS image sensors have three peaks in DCS signals. Peak1 in the distribution corresponds to the dark current induced by process-induced defects during the CMOS image sensor fabrication process. We also found that the DCS spectrum of a CMOS image sensor with hydrocarbon molecular ion implantation substantially decreased by two DCS signal peaks (Peaks 2 and 3) as shown in Fig. 9. ${ }^{(43)}$

Domengie et al. demonstrated the effects of DCS signals in a CMOS image sensor intentionally contaminated with metallic impurities (W and $\mathrm{Au}$ ) and used DLTS to evaluate them. ${ }^{(46)}$ They found that the DCS signal has three peaks. They measured W- and Au-related deep-level defects in the space charge region using DLTS. They understood that the DCS signal peaks were enhanced by metallic-impurity ( $\mathrm{W}$ and $\mathrm{Au}$ )-related deep level defects in the silicon band gap. Russo and coworkers demonstrated the effects of the dark current of a CMOS image sensor intentionally contaminated with metallic impurities $(\mathrm{Cr}, \mathrm{V}, \mathrm{Cu}, \mathrm{Ni}, \mathrm{Fe}, \mathrm{Ti}, \mathrm{Mo}, \mathrm{W}, \mathrm{Al}$, and $\mathrm{Zn}$ ) and used DCS to evaluate them. ${ }^{(47,48)}$ Their results indicate that the Mo, W, Cu, and Ti impurities have greater effects on dark current generation than other metallic impurities. This is because, the $\mathrm{Mo}, \mathrm{W}, \mathrm{Cu}$, and Ti impurities form deep-energy-level defects in the silicon band gap. $^{(11)}$

Therefore, our observation of two DCS signal peaks (Peaks 2 and 3) indicates that metallic impurities form deep-energy-level defects in the device active area (i.e., the photo diode space charge region). These defects can act as generation-recombination centers (G-R centers) in the silicon band gap. Thus, photo diode leakage current increased the number of G-R centers, which increased after metallic impurity contamination during the device fabrication process. DCS signals strongly correlate with photo-diode leakage current. We understand that the two DCS signal peaks originated from the observed metallic-impurity-related defects in the photo diode space charge region. Thus, we assume that the CMOS image sensor with hydrocarbon molecular ion implantation has a high gettering capability during the device fabrication process. 

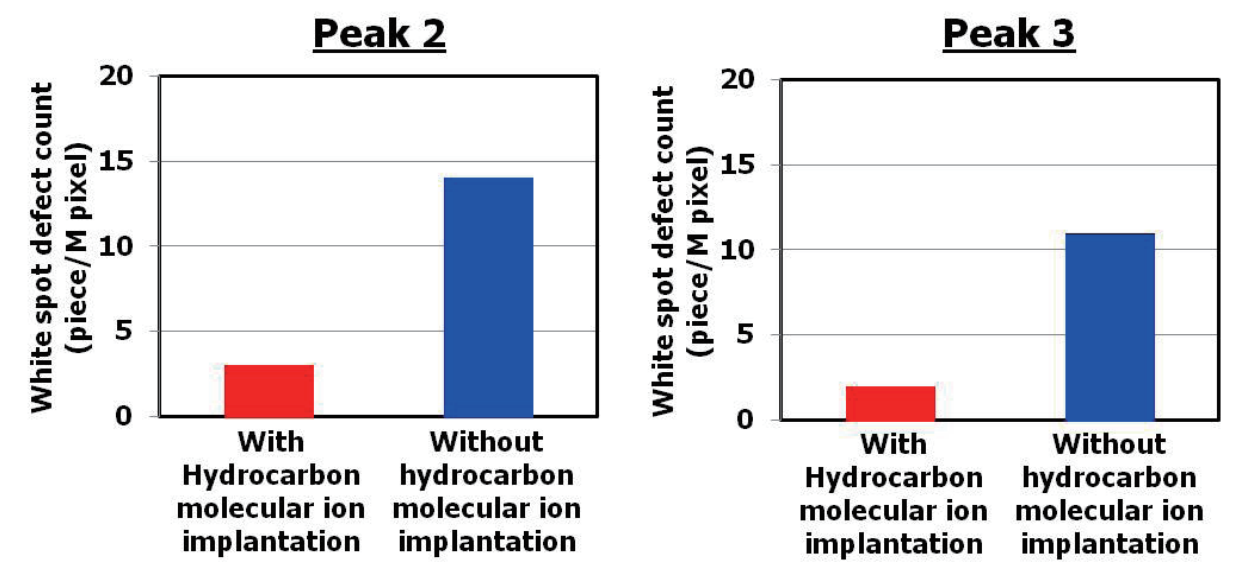

Fig. 9. (Color online) Histograms of white spot defect counts of CMOS image sensors with and without hydrocarbon molecular ion implantation after CMOS image sensor fabrication process. ${ }^{(43)}$

\subsection{TEM observation results of hydrocarbon-molecular-ion-implanted epitaxial silicon wafer before and after the CMOS image sensor fabrication process}

Figure 10 shows the cross-sectional TEM observation results we obtained for the hydrocarbon-molecular-ion-implanted epitaxial silicon wafer after the CMOS device heat treatment. ${ }^{(43)}$ We found that there were hydrocarbon-molecular-ion-implantation-related defects in the hydrocarbon molecular ion implantation projection range. The defects were approximately $5 \mathrm{~nm}$ in size and $1 \times 10^{16} \mathrm{~cm}^{-3}$ in density. No secondary extended defects such as dislocations and dislocation loops were found in the hydrogen molecular ion implantation projection range as shown in Fig. 10.

\subsection{APT observation results of hydrocarbon-molecular-ion-implanted epitaxial silicon wafer after the CMOS image sensor fabrication process}

Figure 11 shows the cross-sectional observation results we obtained for an APT needleshaped specimen prepared using field ion beams from a hydrocarbon-ion-implanted epitaxial silicon wafer after the CMOS image sensor fabrication process. ${ }^{(43)}$ The capping material nickel was used to form the surface position. After preparing a needle-shaped specimen, we measured the three-dimensional impurity distribution in the hydrocarbon molecular ion implantation projection range after the CMOS image sensor fabrication process. Figure 12 shows the APT map of a hydrogen-molecular-ion-implanted epitaxial silicon wafer in the hydrogen molecular ion implantation projection range after the CMOS image sensor fabrication process. The map data indicates that the carbon atoms agglomerate themselves in various forms such as carbon complexes during the CMOS heat treatment. Moreover, oxygen atoms segregated carbon complexes during the CMOS heat treatment. 


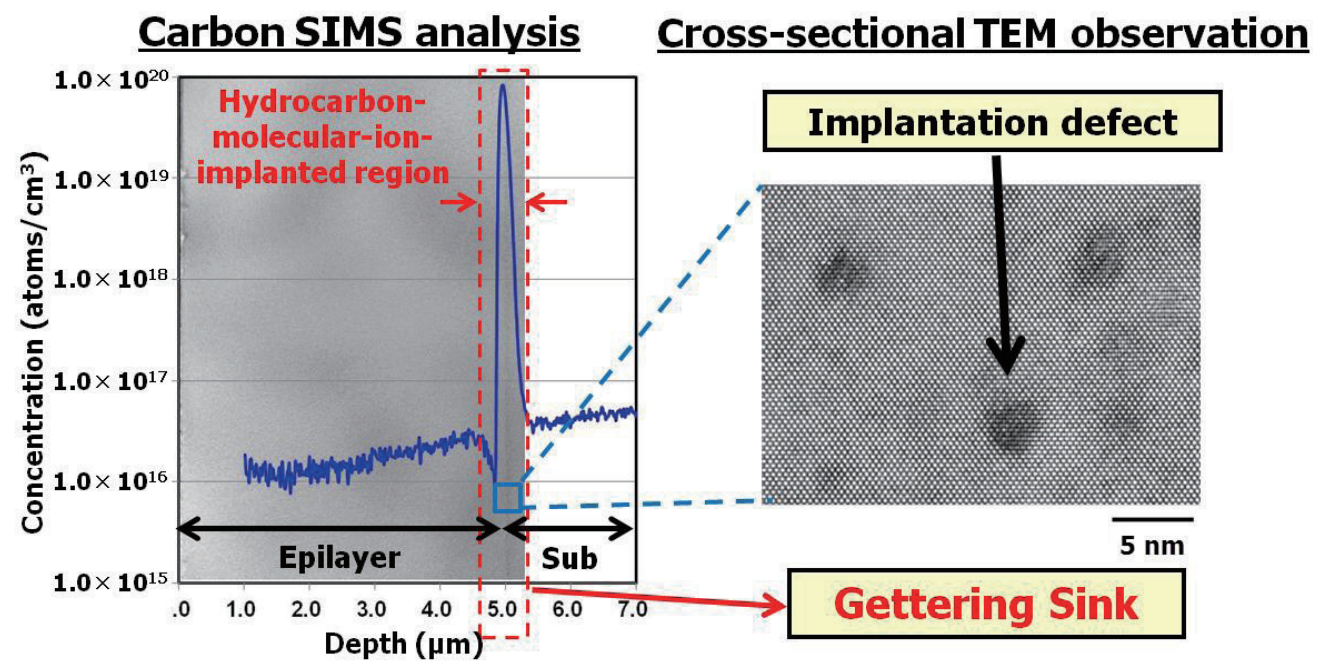

Fig. 10. (Color online) Cross-sectional TEM observation results and SIMS depth profile of carbon in hydrocarbon molecular ion implantation projection range after CMOS image sensor fabrication process. ${ }^{(43)}$

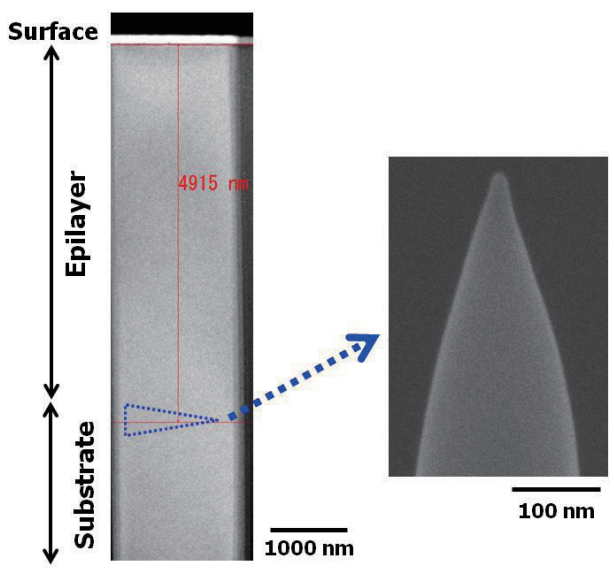

Fig. 11. (Color online) Cross-sectional SEM observation results of needle-shaped specimen for atom probe tomography prepared using field ion beam from hydrocarbon-ion-implanted silicon wafer after CMOS image sensor fabrication process. ${ }^{(43)}$

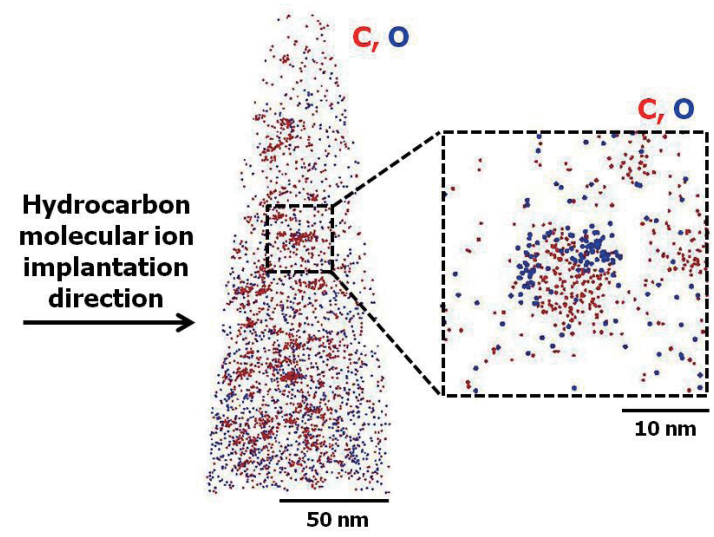

Fig. 12. (Color online) Atom probe tomography map of hydrocarbon molecular ion implantation projection range after CMOS image sensor fabrication process. ${ }^{(43)}$

\section{Discussion}

\subsection{Gettering mechanism of hydrocarbon-molecular-ion-implanted epitaxial silicon wafer}

Why does the CMOS image sensor with hydrocarbon molecular ion implantation has a high gettering capability during the device fabrication process? There are two possible gettering mechanisms that can explain this. 


\subsubsection{Relaxation-induced gettering mechanism ${ }^{(50,51)}$}

Metallic impurities can be gettered by ion-implantation-related defects such as dislocations and dislocation loops. A previous study showed high-energy ion-implantation-induced defects in the projection range. These defects induced strain and stress in the silicon matrix interface. Their effectiveness in gettering impurities is explained by the Cottrell effect, in which the solubility of an impurity atom is greater in the vicinity of secondary extended defects such as dislocations and dislocation loops.

The experimental results we obtained from the TEM observation are shown in Fig. 10. The obtained TEM observation results did not reveal any secondary extended defects in the hydrocarbon molecular ion implantation projection range. However, our previous study results showed that a hydrocarbon-molecular-ion-implanted epitaxial silicon wafer can getter metallic impurities after device heat treatment as shown in Fig. 4. Therefore, the relaxation-induced gettering mechanism cannot account for our experimental results.

\subsubsection{Segregate-induced gettering mechanism ${ }^{(52)}$}

With the segregate-induced gettering mechanism, the solid solubility of metallic impurities in gettering sinks increased substantially more than that for silicon crystal bulk without gettering sinks. Figure 4 shows the SIMS depth profile of metallic impurities in the hydrocarbon molecular ion projection range after metallic impurity diffusion annealing. The metallic impurities are gettered by the hydrocarbon molecular ion projection range. The metallic impurity concentration in the projection range is higher than the solid solubility in silicon crystal bulk. Thus, the experimental results indicate that the hydrocarbon molecular ion projection range forms segregate-induced gettering sinks in silicon crystal bulk.

\subsection{Origin of gettering sinks in hydrocarbon-molecular-ion-implanted epitaxial silicon wafer}

What is the origin of metallic impurity gettering sinks in the hydrocarbon molecular ion implantation projection range? We used APT to observe the hydrogen molecular ion implantation-related defects in the implantation projection range (Fig. 12). ${ }^{(43)}$ The obtained results indicate that the defects form carbon oxygen agglomerates such as carbon complexes in the implantation projection range after the CMOS image sensor fabrication process. We analyzed the APT map of carbon complexes in determining the hydrogen molecular ion implantation range of the carbon complex density and size using Integrated Visualization and Analysis Software (IVAS) from CAMECA. ${ }^{(53)}$ We determined that the carbon complex density is $1 \times 10^{16} \mathrm{~cm}^{-3}$ and that the size is $5 \mathrm{~nm}$. This means that we observed the same hydrogen molecular ion implantation-related defects such as carbon complexes in the hydrogen molecular ion implantation projection range that we did using TEM observation results after the CMOS image sensor fabrication process (see Fig. 10). 
Why are the gettering sinks of carbon complexes in the ion implantation projection range effective for metallic impurity gettering? We assume that these sinks strongly interact with metallic impurities in a hydrocarbon molecular ion implantation projection range.

Shirasawa et al. considered the above the issues from the viewpoint of theoretical solid-state physics. ${ }^{(54-56)}$ They obtained theoretical results by first-principles calculation to determine the binding energies of metallic impurities and their complexes with intrinsic point defects, which were found to be the cause of the effectiveness of hydrogen molecular ion implantation gettering sinks. They indicated that the origin of the metallic impurity gettering sink must be interstitial carbon (Ci) and intrinsic point-defect complexes (carbon self-interstitial clusters, vacancyoxygen pairs, and vacancy-hydrogen pairs).

Furthermore, Okuyama et al. demonstrated that the gettering behavior of hydrogen in the hydrocarbon molecular ion implantation projection range after epitaxial growth can be calculated by technology computer-aided design with a reaction model in which hydrogen binds to a carbon and silicon self-interstitial cluster (Cs-I) ${ }^{(57)}$ This calculation result indicates that a Cs-I cluster is an extremely effective gettering sink for metallic impurities in the hydrocarbon molecular ion implantation projection range.

Therefore, we propose that the hydrocarbon molecular ion implantation projection range must be formed in configurations such as carbon complexes, vacancy-oxygen pairs, and vacancy-hydrogen pairs for effective gettering sinks. We also used APT to observe the hydrocarbon molecular ion implantation projection range. The results we obtained support the hypothesis that the observed carbon complexes are one of the origins of gettering sinks in the hydrocarbon molecular ion implantation projection range after the CMOS device fabrication process. We believe that the segregated-induced gettering sinks originate from carbon complexes in hydrocarbon molecular ion implantation projection in silicon wafers.

\section{Conclusions}

The advanced CMOS image sensor fabrication process involves a number of serious technical issues such as metallic impurity contamination, dark current, and image lag. We consider that these issues require silicon wafer technology solutions using hydrocarbon molecular ion implantation. Accordingly, we developed a hydrocarbon-molecular-ionimplanted epitaxial silicon wafer with a high gettering capability for metal, oxygen, and hydrogen impurities during the CMOS image sensor fabrication process. This novel silicon wafer substantially decreased the white spot defect density and dark current in the CMOS image sensor fabrication line. In conclusion, we believe that such a wafer will be beneficial for advanced CMOS image sensor manufacturing.

\section{Acknowledgments}

The authors would like to express their thanks to Dr. Hisashi Furuya and Mr. Naoki Ikeda for their continuous encouragement extended to our research group concerning fundamental research activities and useful technical discussion with our R\&D team. 


\section{References}

1 T. Kuroda: Essential Principles of Image Sensors (CRC Press, Tokyo, 2014) Chap. 5, p. 55.

2 H. Takahashi: CMOS Image Sensor (Corona, Tokyo, 2012) Chap. 3, p. 123 (in Japanese).

3 K. Graff: Metal Impurities in Silicon-Devices Fabrication (Springer, Berlin/Heidelberg, 2000) p. 20. https:// doi.org/10.1007/978-3-642-57121-3

4 C. Claeys and E. Simoen: Metal Impurities in Silicon and Germanium-Based Technologies (Springer, Switzerland, 2018) Chap. 7, p. 332. https://doi.org/10.1007/978-3-319-93925-4

5 E. R. Weber: Appl. Phys. A 30 (1983) 1. https://doi.org/10.1007/BF00617708

6 A. A. Istratov and E. R. Weber: Appl. Phys. A 66 (1998) 123. https://doi.org/10.1007/s003390050649

7 L. Jastrzebski, R. Soydan, G. W. Cullen, W. N. Henry, and S. Vecrumba: J. Electrochem. Soc. 134 (1987) 214. https://doi.org/10.1149/1.2100410

8 A. L. Rotondara, T. Q. Hurd, A. Kaniava, J. Vanhellemont, E. Simoen, M. M. Heyns, C. Claeys, and G. Brown: J. Electrochem. Soc. 143 (1996) 3014. https://doi.org/10.1149/1.2100410

9 K. W. Lee, J.-C. Bea, T. Fukushima, T. Tanaka, and M. Koyanagi: J. Electrochem. Soc. 158 (2011) H795. https://doi.org/10.1149/1.3597317

10 D. Gilles, E. R. Weber, and S. Hahn: Phys. Rev. Lett. 64 (1990) 196. https://doi.org/10.1103/PhysRevLett.64.196

11 M. Aoki, A. Hara, and A. Ohsawa: J. Appl. Phys. 72 (1992) 895. https://doi.org/10.1063/1.351764

12 F. Shimura: Semiconductor Silicon Crystal Technology (Academic Press, Cambridge, MA, 1989) p. 359.

13 H. Wong, N. W. Cheung, and P. K. Chu: Appl. Phys. Lett. 52 (1988) 889. https://doi.org/10.1063/1.99233

14 H. Wong, N. W.Cheung, P. K. Chu, J. Liu, and J. W. Mayer: Appl. Phys. Lett. 52 (1988) 1023. https://doi. org/10.1063/1.99263

15 T. Kuroi, Y. Kawasaki, S. Komori, K. Fukumoto, M. Inoshi, K. Tsukamoto, H. Shinyashiki, and T. Shingyouji: Jpn. J. Appl. Phys. 32 (1993) 303. https://doi.org/10.1143/JJAP.32.303

16 A. Agarwal, K. Christensen, D. Venable, D. M. Maher, and G. A. Rozgonyi: Appl. Phys. Lett. 69 (1996) 3899. https://doi.org/10.1063/1.117563

17 O. Kononchuk, R. A. Brown, Z. Radzimiski, and G. A. Rozgonyi: Appl. Phys. Lett. 69 (1996) 4203. https://doi. org/10.1063/1.116986

18 R. A. Brown, O. Kononchuk, I. Bondarenko,A. Romanowski, Z. Radzzimski, and G. A. Rozgonyi: J. Electrochem. Soc. 144 (1997) 2872. https://doi.org/10.1149/1.1837910

19 S. V. Koveshikov and G. A. Rozgonyi: J. Appl. Phys. 84 (1998) 3078. https://doi.org/10.1063/1.368462

20 S. R. Shortes, W. W. Chan, W. C. Rhines, J. B. Barton, and D. R. Collins: IEDM Tech. Dig. (1973) p. 415.

21 V. Goiffong, P. Magnan, G. P. Martin, C. Virmontosiv, and M. Gaillarding: IEEE Electron Device Lett. 32 (2011) 773.

22 E. H. Nicollian and J. R. Brews: MOS (Metal Oxide Semiconductor Physics and Technology) (Wiley, New York, 1982) p. 823.

23 J. L. Regolini, D. Benoit, and P. Morin: Microelectron. Reliab. 47 (2007) 739. https://doi.org/10.1016/ j.microrel.2007.01.067

24 T. Kadono and K. Kurita: Japan Patent 5673811 (2015).

25 K. Kurita: Oyo Buturi 84 (2015) 628 (in Japanese).

26 K. Kurita, T. Kadono, R. Okuyama, R. Hirose, A. Onaka-Masada, Y. Koga, and H. Okuda: Jpn. J. Appl. Phys. 55 (2016) 121301. https://doi.org/10.7567/JJAP.55.121301

27 R. Okuyama, T. Kadono, A. Masada, R. Hirose, Y. Koga, H. Okuda, and K. Kurita: Jpn. J. Appl. Phys. 56 (2017) 025601. https://doi.org/10.7567/JJAP.56.025601

28 K. Kurita, T. Kadono, R. Okuyama, R. Hirose, A. Onaka-Masada, Y. Koga, and H. Okuda: IEEE Electron Device Technology and Manufacturing Conf., Toyama 6M-4 (2017) 105. https://doi.org/10.1109/ EDTM.2017.7947538

29 R. Okuyama, S. Shigematsu, R. Hirose, A. Masada, T. Kadono, Y. Koga, H. Okuda, and K. Kurita: Phys Status Solidi C 14 (2017) 1700036. https://doi.org/10.1002/PSSC.201700036

30 K. Kurita, T. Kadono, R. Okuyama, S. Shigematsu, R. Hirose, A. Onaka-Masada, Y. Koga, and H. Okuda, Phys. Status Solidi A 214 (2017) 1700216. https://doi.org/10.1002/PSSA.201700216

31 I. Yamada and J. Matsuo: Oyo Buturi 66 (1997) 559 (in Japanese).

32 I. Yamada, J. Matsuo, N. Toyoda, and A. Kirkpatric: Mater. Sci. Eng. R34 (2001) 231. https://doi.org/10.1016/ S0921-5093(96)10358-0

33 M. Tanjyo, N. Hamamoto, T. Nagayama, S. Umisedo, Y. Koga, N. Maehara, H. Une, T. Matsumoto, N. Nagai, and J. O. Borland: ECS Trans. 18 (2009) 1059. https://doi.org/10.1149/1.3096572

34 T. F. Kelly and M. K. Miller: Rev. Sci. Instrum. 78 (2007) 031101. https://doi.org/10.1063/1.2709758 
35 B. Chatterjee and S. A. Ringel: J. Appl. Phys. 77 (1995) 3885. https://doi.org/10.1063/1.358567

36 S. J. Pearton and A. J. Tavendale: Phys. Rev. B 26 (1982) 7105. https://doi.org/10.1103/PhysRevB.26.7105

37 S. J. Pearton, J. W. Corbet, and T. S. Carrbett: Appl. Phys. A 43 (1987) 153. https://doi.org/10.1007/BF00615975

38 S. J. Pearton, J. W. Corbett, and M. Stavola: Hydrogen in Crystalline Semiconductors (Springer-Verlag, New York, 1991). https://doi.org/10.1007/978-3-642-84778-3

39 W. C. Mccolgin, J. P. Lavine, and C. V. Stancampiano: Mat. Res. Soc. Symp. Proc. 378 (1995) 713.

40 W. C. Mccolgin, J. P. Lavine, and C. V. Stancampiano: Mat. Res. Soc. Symp. Proc. 442 (1997) 187.

41 D. V. Lang: J. Appl. Phys. 45 (1974) 3023. https://doi.org/10.1063/1.1663719

42 D. K. Schroder: Semiconductor Material and Device Characterization (Wiley, New York, NJ, 1998) 2nd ed.

43 K. Kurita, T. Kadono, R. Okuyama, R. Hirose, A. Onaka-Masada, Y. Koga, and H. Okuda: 22nd Int. Conf. Ion Implantation Technology (IIT, 2018) 95.

44 H.Takahashi, M. Kinochita, K. Morita, T. Shirai, T. Sato, T. Kimura, H. Yuzurihara, and S. Inoue: ISSCC Dig. Tech. Papers (2004) 78.

45 M. Mori, M. Katsuno, S. Kasuga, T. Murata, and T. Yamaguchi: ISSCC Dig. Tech. Papers (2004) 80.

46 F. Domengie, J. L. Regolini, and D. Bauza: J. Electr. Mater. 39 (2010) 625. https://doi.org/10.1007/s11664-010$1212-6$

47 F. Russo, G. Moccia, G. Nardone, R. Alfonsetti, G. Polsinelli, A. D’Angelo, A. Patacchiola, M. Liverani, P. Pianezza, T. Lippa, M. Carlini, M. L. Polignanol, I. Mica1, E. Cazzinil, M. Ceresoli1, and D. Codegonil: Solid-State Electron. 91 (2014) 91. https://doi.org/10.1016/j.sse.2013.10.011

48 F. Russo, G. Nardone, M. L. Polignano, A. D’Ercole, F. Pennella, M. D. Felice, A. D. Monte, A. Matarazzo, G. Moccia, G. Polsinelli, A. D’angelo, M. Liverani, and F. Irrera: ECS J. Solid State Sci. Technol. 6 (2017) 217. https://doi.org/10.1149/2.0101705jss

49 T. F. Kelly and M. K. Miller: Rev. Sci. Instrum 78 (2007) 031101. https://doi.org/10.1063/1.2709758

50 J. S. Kang and D. K. Schroder: J. Appl. Phys. 65 (1989) 2974. https://doi.org/10.1063/1.342714

51 J. L. Benton, P. A. Stolk, D. J. Eaglesham, D. C. Jacobson, J. Y. Cheng, J. M. Poate, N. T. Ha, T. E. Haynes, and S. M. Myers: J. Appl. Phys. 80 (1996) 3275. https://doi.org/10.1063/1.363236

52 L. Baldi, G. F. Cerofolini, G. Ferla, and G. Frigerio: Phys. Status Solidi 48 (1978) 525.

53 S. Shigematsu, R. Okuyama, R. Hirose, A. Masada, T. Kadono, Y. Koga, H. Okuda, and K. Kurita: The 19th Scientific Int. Symp. SIMS and Related Techniques Based on Ion-Solid Interactions (2017) 22.

54 S. Shirasawa, K. Sueoka, T. Yamaguchi, and K. Maekawa: J. Electrochem. Soc. 4 (2015) 351. https://doi. org/10.1149/2.0051509jss

55 S. Shirasawa, K. Sueoka, T. Yamaguchi, and K. Maekawa: Ext. Abstr. (76th Autumn Meet. 2015), Japan Society of Applied Physics and Related Societies, 14p-PB7-2 (2015).

56 S.Shirasawa, K.Sueoka, T. Yamaguchi, and K. Maekawa: Mater. Sci. Semicond. Process 44 (2016) 13. https:// doi.org/10.1016/j.mssp.2016.01.001

57 R. Okuyama, S. Shigematsu, R. Hirose, A. Masada, T. Kadono, Y. Koga, H. Okuda, and K. Kurita: Phys. Status Solidi C 14 (2017) 1700036. https://doi.org/10.1002/pssc.201700036 


\section{About the Authors}

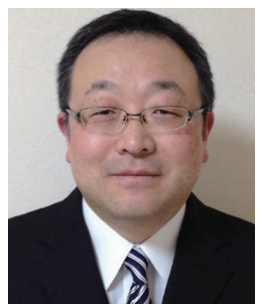

Kazunari Kurita received his B.E. degree in electronics engineering from Akita University, Akita, Japan, in 1991 and his M.E. degree in electronics engineering from Tohoku University, Sendai, Miyagi, Japan, in 1993. He received his Ph.D. degree in electrical and electronics engineering from Kanagawa Institute of Technology (KIT), Atsugi, Kanagawa, Japan, in 2002. From 1993 to 2001, he was a research member of Central Research Institute of Mitsubishi Materials Corporation, Omiya Saitama, Japan. In 2002, he joined the SUMCO Corporation, Tokyo, Japan. He invented the novel gettering technology for advanced CMOS image sensors using a hydrocarbon molecular ion implantation technique. He has published 30 peer-reviewed papers covering gettering technology for CMOS image sensor and holds more than 150 Japan and U.S. patents. His current research focus includes the silicon wafer gettering process and the development of metallic impurity contamination analysis techniques such as deep-level transient spectroscopy (DLTS) and microwave photoconductance decay lifetime measurement (Microwave-PCD) techniques. Since 2016, he has been a General Manager of the R\&D division in SUMCO Corporation. He was a recipient of an honorary award, the 23rd SSSJ Technical Award, from the Surface Science Society of Japan in 2017. (k-kurita@sumcosi.com)

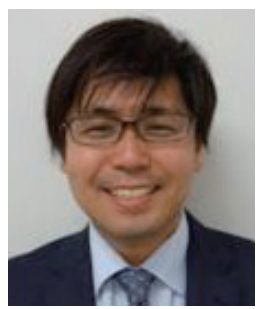

Takeshi Kadono received his B.E. degree in materials physics and engineering and his M.E. degree in applied physics from Miyazaki University, Japan, in 2007 and 2009, respectively. In 2009, he joined the SUMCO Corporation, Tokyo, Japan. His current work involves the product design development of hydrocarbon-molecular-ion-implanted silicon wafers. (tkadono@sumcosi.com)

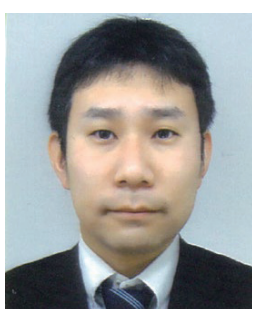

Ryosuke Okuyama received his B.E. degree in materials science and engineering and his M.E. degree in materials physics and chemistry from Kyushu University, Fukuoka, Japan, in 2006 and 2008, respectively. From 2009 to 2012, he was a researcher in Nano-SOI Process Laboratory, Hanyang University in Korea. His main research interests include the kinetic analysis of the hydrogen diffusion behavior and passivation effect in hydrocarbonmolecular-ion-implanted wafers for advanced CMOS image sensors. (rokuyama@sumcosi.com) 


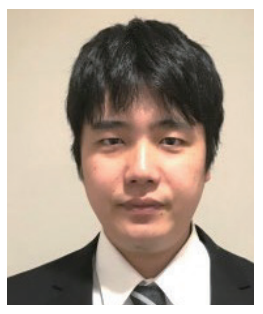

Satoshi Shigematsu was born in Chikushino, Fukuoka, Japan, in 1990. He received his B.Sc. and M.Sc. degrees in physics from Kagoshima University, Kagoshima, Japan, in 2014 and 2016, respectively. From 2016, he studies the gettering behavior of molecular-ion-implanted silicon wafers in atomic scale using atom probe tomography in SUMCO Corporation, Tokyo, in Japan. (sshigema@sumcosi.com)

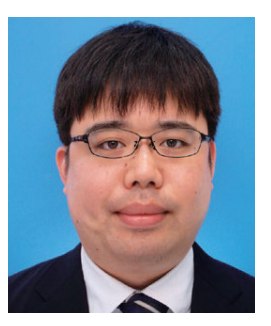

Ryo Hirose was born in Kyoto, Japan. He received his B.E. degree in chemical science and engineering and his M.S. degree in chemistry from Osaka University, Japan, in 2012 and 2014, respectively. In April 2017, he joined the Department of Nuclear Engineering at Kyoto University, where he is currently pursuing his Ph.D. degree. In 2014, he joined SUMCO Corporation, Tokyo, Japan. He works on the development of a new gettering technique for advanced CMOS image sensors using multielement molecular ion implantation. (rhirose@sumcosi.com)

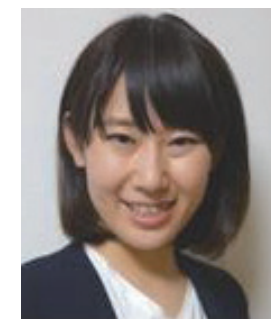

Ayumi Onaka-Masada was born in Himeji, Hyogo, Japan, in 1985. She received her B.E. degree in applied chemistry from the University of Hyogo, Hyogo, Japan, in 2008 and her M.S. degree in chemistry from Osaka University, Osaka, Japan, in 2010. She is currently pursuing her Ph.D. degree at Okayama Prefectural University, Okayama, Japan. In 2010, she joined SUMCO Corporation in Japan. She worked in the research and development group in the area of metallic impurity contamination analysis in silicon wafers using deep-level transient spectroscopy (DLTS). She currrently studies gettering technology for advanced CMOS image sensors.

(aonaka@sumcosi.com)

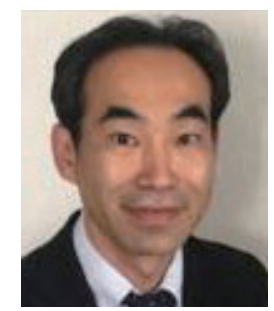

Yoshihiro Koga was born in Fukuoka, Japan, in 1971. He received his B.E. and M.E. degrees in the Department of Electrical and Electronic Engineering from Nagoya Institute of Technology in 1995 and 1998, respectively. From 1998 to 2006, he developed power devices at Oki Electric Industry Co., Ltd. He has been developing semiconductor materials for CMOS image sensors and power devices at SUMCO Corporation. (ykoga4@sumcosi.com)

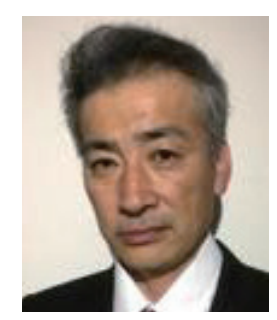

Hidehiko Okuda was born in Isahaya, Nagasaki, Japan, in 1966. He received his B.E. degree in materials engineering from Nagasaki University, Nagasaki, Japan, in 1993. In 1993, he joined SUMCO Corporation. He currently works on the development of an annealing process for high-quality Si wafers. (hokuda@sumcosi.com) 